\title{
Characterization of biliary confluence angle in Asian population
}

\author{
Eman Abdul Rahman Mohammed Altai ${ }^{1}$, Caroline Edward Ayad ${ }^{2}$, Elsafi Ahmed Abdalla ${ }^{2}$ \\ ${ }^{1}$ Radiology Department, Dr Soliman Fakeeh Hospital-Al-Medina, Qurban, Saudi Arabia \\ ${ }^{2}$ Sudan University of Science and Technology, College of Medical Radiological Science, Khartoum, Sudan
}

\section{Email address:}

emanabdlrhman@yahoo.com (E. A. R. M. Altai), Carolineayad@yahoo.com (C. E. Ayad), carolineyad@sustech.edu (C. E. Ayad), dr.elsafi@gmail.com (E. A. Abdalla),dr.elsafi@sustech.edu (E. A. Abdalla)

\section{To cite this article:}

Eman Abdul Rahman Mohammed Altai, Caroline Edward Ayad, Elsafi Ahmed Abdalla. Characterization of Biliary Confluence Angle in Asian Population. American Journal of Health Research. Vol. 2, No. 2, 2014, pp. 73-77. doi: 10.11648/j.ajhr.20140202.17

\begin{abstract}
This study aimed to evaluate the morphological alterations in the biliary confluence angle of Asian population, describing the most frequent MR cholangiopancreatography (MRCP) findings, evaluating the confluence angle in the cases of dilated and normal ducts, and to correlate the angle with the patients age, gender, nationality, height, weight and body mass index (BMI). The study was done in Suleiman Fakeh Hospital in Jeddah KSA, in the period from March 2011 up to May 2012. The sample was drawn from patients in both gender referred to MRCP imaging with different indications and was classified into two groups, the first group was patients with normal biliary ducts, the second group was patients with dilated biliary ducts . All examinations were done using MRI 1.5 Tesla, Siemens Avanto 2010, single shot fast spin echo (SSFSE). Protocol using coronal oblique images was applied where the angle was measured. Results showed that the most common MRCP findings were: distended gall bladder, gall stone, cholecystitis, cholecystectomy, liver cirrhosis, hepatomegaly, hepatic lesion, pancreatitis ,and pancreatic lesion. MRCP can evaluate the biliray confluence angle in dilated and normal ducts. No significant relation was found between the biliary confluence angle and the selected variables in both normal and dilated ducts, but a significant relation was detected with the age in patients with normal biliary ducts. Dependency upon the biliary confluence angle is not benifectual for diagnosis or prediction of diseases. A new equation for predicting the biliary confluence angle with the known Asian ages was established.
\end{abstract}

Keywords: Bile Ducts, Cholangiopancreatography, MRCP

\section{Introduction}

MR cholangiopancreatography (MRCP) is a new technique which has replaced the use of diagnostic endoscopic retrograde cholangiopancreatography (ERCP). MRCP is a heavily $\mathrm{T}_{2}$-weighted MR sequence that presents the fluid containing ducts noninvasively, avoiding any complications associated with ERCP. [1]

The biliary confluence angle is the angle between the right and left hepatic ducts; the importance of the angle is that the widening of the angle may be relevant when a mass, cholangitis or other diseases are located in this area, but the width of this angle may also be wider in some normal individuals .[2]

Many Alterations of the biliary tree and confluence angle may happen and is needed to be investigated by means of a noninvasive method, leading to enhance the findings in different cases, to our knowledge no angle was detected as reference for Asian populations. So, the present study aimed at corroborating the MRCP as a new imaging method, by evaluating the morphological alterations seen in the biliary confluence angle of Asian residents, describing the most frequent MRCP findings, evaluating the confluence angle in the cases of dilated ducts with other MRCP findings and normal ducts with abnormal results, as well as correlating the biliary confluence angle with the patients demographic data including age, gender, Body Mass Index (BMI), race ,height and weight in addition to study the ability of prediction the disease causes when the angle is known .

\section{Materials and Methods}

\subsection{Area, Duration and Sample Selection}

The research was done in Suleiman Fakeh Hospital in Jeddah KSA with approval and agreement to provide all data for the study in the period from March 2011 up to May 2012. 
The sample was drawn from patients referred to MRCP imaging with different clinical indications and was classified into two groups, the first group included 137 patients, $76(55.5 \%)$ were males and $61(44.5 \%)$ were females they were subjects with normal biliary ducts with different MRCP findings as distended gall bladder, gall stone, cholecystitis,cholecystectomy, liver cirrhosis, hepatomegaly, hepatic lesion, pancreatitis ,and pancreatic lesion. The second group included 28patients $18(64.3 \%)$ were females and $10(35.7 \%)$ were males with dilated biliary ducts and associated MRCP findings.

\subsection{MRCP Technique}

All the examinations were done on patients fasting for about 6 hours using MRI Siemens Avanto 2010 strength 1.5 Tesla closed MRI machine with super conductive coil, the selected parameters were, Flip angle(170-180), Field of view FOV(100),TR (5122)ms,TE(678)ms, Matrix medium matrix $384 \backslash 384$, the applied Protocol is single shot fast spin echo (SSFSE) coronal oblique $\mathrm{T}_{1}$ weighted, $\mathrm{T}_{2}$ weighted , $\mathrm{T}_{1}$ with gadolinium contrast enhancement images were obtained. The angle was measured using machine measurement tools by drawing two lines running within the origins of both RT and LT hepatic ducts and crossing at biliary confluence angle on coronal oblique images. The data were analyzed using SPSS Version16, ANOVA independent variable, mean and standard deviation, frequency and percentage.

\section{Results}

\subsection{Group1}

All the 137 patients showed Normal biliary ducts and other MRCP findings. The Descriptive Statistics of the sample showed their mean values of BMI was (78.3 \pm 15.2$)$, Age $(45.5 \pm 16.9)$, Weight $(78.3 \pm 15.2)$, Height $(164.4 \pm$ $8.1)$, Biliary angle $(83.4 \pm 23.5), 89(65 \%)$ were Saudian and $48(35 \%)$ were non Saudi population including Indian, Bangladesh and Pakistani.

Table (1). Distribution of the study sample with normal biliary tree according to Diagnosis/Findings by MRCP.

\begin{tabular}{lll}
\hline $\begin{array}{l}\text { Normal Biliary Tree and MRCP } \\
\text { Diagnosis/findings }\end{array}$ & Frequency & Percentage\% \\
\hline Normal Findings & 40 & $29.2 \%$ \\
Cholecystectomy & 5 & $3.6 \%$ \\
Cholecystitis & 6 & $4.4 \%$ \\
Liver Cirrhosis & 2 & $1.5 \%$ \\
Distended Gall Bladder & 6 & $4.4 \%$ \\
Hepatomegaly & 8 & $5.8 \%$ \\
Mirrizzi Syndrome & 1 & $0.7 \%$ \\
Pancreatic Lesion & 6 & $4.4 \%$ \\
Pancreatitis & 9 & $6.6 \%$ \\
Gall Stone & 54 & $39.4 \%$ \\
Total & 137 & $100.0 \%$ \\
\hline
\end{tabular}

Table (2). Biliary angle measured in Normal Biliary Tree \& MRCP findings P-value 0.163 .

\begin{tabular}{llll}
\hline Diagnosis & Mean $\pm \boldsymbol{S ~ D}$ & Maximum & Minimum \\
\hline Normal Finding & $85.6 \pm 24.3$ & 155.6 & 41.4 \\
Cholecystectomy & $81.1 \pm 18.6$ & 98.0 & 52.4 \\
Cholecystitis & $97.0 \pm 11.6$ & 111.3 & 80.6 \\
Liver Cirrhosis & $60.3 \pm 20.0$ & 74.4 & 46.1 \\
Distended GB & $76.4 \pm 25.8$ & 101.7 & 36.4 \\
Hepatomegaly & $96.6 \pm 26.6$ & 142.2 & 61.7 \\
Mirrizzi Syndrome & $103.9 \pm 0.0$ & 103.9 & 103.9 \\
Pancreatic Lesion & $63.8 \pm 16.6$ & 95.4 & 52.4 \\
Pancreatitis & $77.7 \pm 17.4$ & 100.7 & 44.5 \\
Gall Stone & $82.8 \pm 24.0$ & 115.8 & 36.4 \\
Total & $83.4 \pm 23.5$ & 155.6 & 36.4 \\
\hline
\end{tabular}

Table (3). Biliary angle measured in patients with normal duct related to Gender.

\begin{tabular}{llll}
\hline Gender & Mean \pm S D & Maximum & Minimum \\
\hline Male & $80.1 \pm 21.2$ & 142.2 & 36.4 \\
Female & $87.4 \pm 25.7$ & 155.6 & 38.1 \\
Total & $83.4 \pm 23.5$ & 155.6 & 36.4 \\
$P$-Value $=0.071$ & & \\
\hline
\end{tabular}

Table (4). Biliary angle measured in patients with normal duct related to Nationality.

\begin{tabular}{llll}
\hline Nationality & Mean \pm S D & Maximum & Minimum \\
\hline Saudi & $83.7 \pm 21.8$ & 130.4 & 36.4 \\
Non Saudi & $81.98 \pm 33.9$ & 128.1 & 52.3 \\
Total & $83.4 \pm 23.5$ & 155.6 & 36.4 \\
$p$-Value $=0.958$ & & & \\
\hline
\end{tabular}

Table (5). Biliary angle measured in patients with normal duct related to BMI, Age, Weight and Height.

\begin{tabular}{lllll}
\hline Biliary angle & BMI & Age & Weight & Height \\
\hline Correlation Coefficient & -0.041 & -0.193 & -0.041 & 0.041 \\
P-value & 0.632 & $0.024^{*}$ & 0.632 & 0.632 \\
\hline
\end{tabular}

* Significant at $\mathrm{P}$-value $<0.05$.

Table (6). Regression Analysis of Biliary angle measured in patients with normal duct \& Age.

\begin{tabular}{llll}
\hline \multirow{2}{*}{ Model } & \multirow{2}{*}{ B } & \multicolumn{2}{c}{$\mathbf{9 5 . 0 \%}$ Confidence Interval for B } \\
& & Lower Bound & Upper Bound \\
\hline (Constant) & 95.601 & 84.285 & 106.917 \\
Age & -0.269 & -0.502 & -0.036 \\
\hline
\end{tabular}

Equation: Biliary angle $=95.6-0.269$ Age, Determination Coefficient $\left(\mathrm{R}^{2}\right)$ $=3.7 \%$ : Interpretation : $(95.6)$ is mean Biliary angle when (age $=0)$. The mean of Biliary angle decrease by $(0.269)$ when the age Increases by one year. The determination coefficient means the $(3.7 \%)$ variation occurs in biliary angle from the age.

Table (7). Comparison between Biliary confluence angle in Normal \& Dilatede Biliary ducts.

\begin{tabular}{llll}
\hline Type & N & Mean \pm S D & P-value \\
\hline Normal Biliary ducts & 137.0 & $83.4 \pm 23.5$ & \multirow{2}{*}{0.376} \\
Dilated Biliary ducts & 28.0 & $79.1 \pm 20.2$ & \\
\hline
\end{tabular}

\subsection{Group2}

28 patients with Dilated Biliary ducts were included, $18(64.3 \%)$ were females and 10(35.7\%) were males and had associated MRCP findings. The Descriptive Statistics of the sample showed their mean values of BMI was $(60.8 \pm 18.4)$, 
Age $(55.9 \pm 21.8)$, Weight $(60.8 \pm 18.4)$, Height $(154.9 \pm$ $10.3)$, Biliary angle $(79.1 \pm 20.2), 20(71 \%)$ were Saudian and $8(28.6 \% \%)$ were non Saudi population including Indian, Bangladesh and Pakistani

Table (8). Distribution of the study sample (group 2) with dilated biliary ducts according to (Diagnosis MRCP findings)

\begin{tabular}{lll}
\hline Dilated Biliary duct/Diagnosis & Frequency & Percentages\% \\
\hline Cholecystectomy & 5 & $17.9 \%$ \\
Normal Findings & 9 & $32.1 \%$ \\
Distended Gall Bladder & 4 & $14.3 \%$ \\
Gall Stone & 10 & $35.7 \%$ \\
Total & 28 & $100.0 \%$ \\
\hline
\end{tabular}

Table (9). Biliary angle measured in patients with dilated biliary tree\& MRCP Diagnosis

\begin{tabular}{llll}
\hline Diagnosis & Mean \pm S D & Maximum & Minimum \\
\hline Cholecystectomy & $85.2 \pm 28.1$ & 134.8 & 65.5 \\
Normal Findings & $75.0 \pm 22.7$ & 104.8 & 38.8 \\
Distended GB & $67.9 \pm 15.5$ & 88.5 & 55.5 \\
Gall Stone & $84.2 \pm 14.5$ & 108.9 & 65.0 \\
Total & $79.1 \pm 20.2$ & 134.8 & 38.8 \\
\hline
\end{tabular}

Table (10). Biliary angle in patients with dilated ducts classified according to gender Value $=0.830$

\begin{tabular}{llll}
\hline Gender & Mean \pm S D & Maximum & Minimum \\
\hline Male & $78.0 \pm 15.4$ & 105.7 & 63.8 \\
Female & $79.7 \pm 22.8$ & 134.8 & 38.8 \\
Total & $79.1 \pm 20.2$ & 134.8 & 38.8 \\
\hline
\end{tabular}

Table (11). Biliary angle measured in patients with dilated ducts related to Nationality

\begin{tabular}{llll}
\hline Nationality & Mean \pm S D & Maximum & Minimum \\
\hline Saudi & $79.4 \pm 21.7$ & 134.8 & 38.8 \\
Non Saudi & $78.4 \pm 16.9$ & 108.9 & 65.0 \\
Total & $79.1 \pm 20.2$ & 134.8 & 38.8 \\
$p$-Value $=0.913$ & & & \\
\hline
\end{tabular}

Table (12). Biliary angle measured in patients with dilated duct related to BMI, Age, Weight and Height

\begin{tabular}{lllll}
\hline Biliary angle & BMI & Age & Weight & Height \\
\hline Correlation Coefficient & -0.140 & -0.216 & -0.140 & 0.156 \\
P-value & 0.477 & 0.270 & 0.477 & 0.429 \\
\hline
\end{tabular}

3.3. Comparison between the Samples with Normal bile ducts and dilated bile ducts and MRCP findings

Table(13). Normal biliary tree \& dilated biliary tree with normal MRCP Findings

\begin{tabular}{llcc}
\hline Diagnosis & N & Mean \pm S D & P-value \\
\hline $\begin{array}{l}\text { Normal Biliary Tree/ Normal } \\
\text { MRCP Finding }\end{array}$ & 40.0 & $85.6 \pm 24.3$ & \\
$\begin{array}{l}\text { Dilated Biliary /Normal } \\
\text { MRCP findings }\end{array}$ & 9.0 & $75.0 \pm 22.7$ & 0.241 \\
\hline
\end{tabular}

Table(14). Normal and dilated Biliary tree with pathological MRCP Findings

\begin{tabular}{llcl}
\hline Diagnosis & N & Mean \pm S D & P-value \\
\hline $\begin{array}{l}\text { Normal Biliary } \\
\text { withcholecystectomy }\end{array}$ & 5.0 & $81.1 \pm 18.6$ & \\
$\begin{array}{l}\text { Biliary dilatation with } \\
\text { cholecystectomy }\end{array}$ & 5.0 & $85.2 \pm 28.1$ & 0.796 \\
\hline
\end{tabular}

\begin{tabular}{lccc}
\hline Diagnosis & N & Mean \pm S D & P-value \\
\hline $\begin{array}{l}\text { Normal biliary with } \\
\text { distended gall bladder }\end{array}$ & 6.0 & $76.4 \pm 25.8$ & \\
$\begin{array}{l}\text { Biliary dilatation with } \\
\text { distended gall bladder }\end{array}$ & 4.0 & $67.9 \pm 15.5$ & 0.572 \\
$\begin{array}{l}\text { Normal biliary with gall } \\
\text { stone }\end{array}$ & 54.0 & $82.8 \pm 24.0$ & 0.854 \\
$\begin{array}{l}\text { Biliary dilatation with gall } \\
\text { stone }\end{array}$ & 10.0 & $84.2 \pm 14.5$ & \\
\hline
\end{tabular}

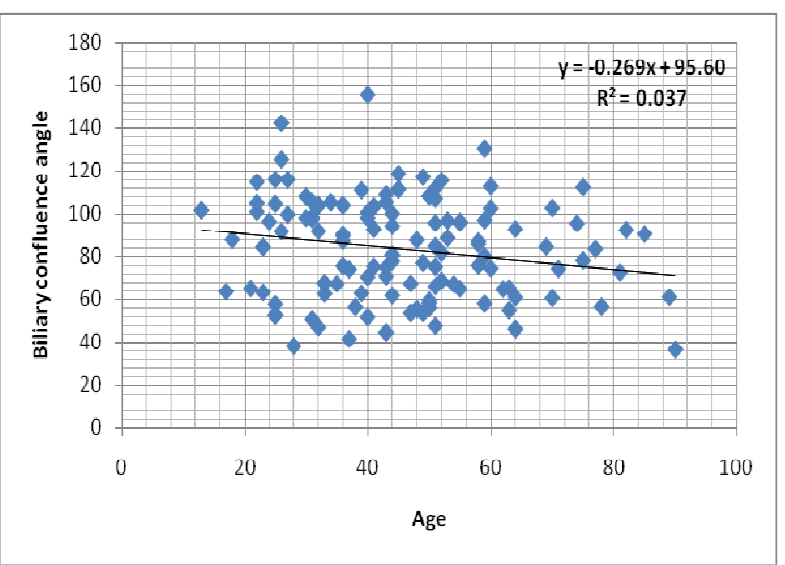

Figure 1. A scatter plot diagram shows the linear relationship between the age and biliary confluence angle, the angle was decreased as the age increased.

\section{Discussion}

Anatomically and during the embryological development, intra hepatic bile ducts line with the portal vein branches. The bile ducts follow the course of the portal vein divisions. $[3,4]$ From the morphological point of view regarding biliary tree alterations, it is possible that there is an association with diseases as fibrosis, intrinsic microscopic alterations of the biliary ducts, portal vessels ectaticity which affect the adjacent biliary tract. [5] The importance of biliary confluence angle is that it may be relevant to different diseases as well as its surgical importance in liver transplantation. [6-9]

Magnetic resonance cholangiopancreatography (MRCP), is an accepted imaging methods for the biliary system which present the possibility of acquiring anatomic cross-sectional information through imaging examination.[10]The use of this method helps in the diagnosis of different diseases. $T_{1}$ and $\mathrm{T}_{2}$ weighted MR images were performed as part of routine MR examination. These images are helpful for diagnosing causes of biliary obstruction and pathology other than stones, gadolinium- enhanced $\mathrm{T}_{1}$-weighted imaging is useful in the setting of suspected neoplasm or inflammatory diseases, including cholangitis, cholecystitis, and pancreatitis, so these protocols were applied in our study in order to improve the visualization of biliary ducts by increasing the contrast between fluids and other structures .As the bile has high water content. It appears bright with its long $\mathrm{T}_{2}$ relaxation times; whereas parenchyma tissues are lower in signal intensity. [11]

This study divided the sample into two groups one with normal biliary duct and the second group with dilated ducts. 
Patients in the first group of normal biliary ducts was studied properly by measuring their weight, height, BMI and age ,this group included different Asian populations in both genders ,the Saudi were more than non Saudi patients, the patients MR images were diagnosed by two expertise radiologists and the findings were noted as normal hepatic ducts without dilatation associated with different MRCP findings including: normal biliary system, cholecystitis, liver cirrhosis,distended gall bladder, hepatomegaly, Mirrizzi syndrome, pancreatic lesion, pancantrisitis ,cholecystectomy as seen in table[1]. The confluence angles were presented as mean and standard deviation with maximum and minimum values as shown in table [2]. When the biliary confluence angle is normal without any dilatation; no significant relation was found in biliary angle measurements and associated diseases as diagnosed by MR imaging. The mean biliary confluence angle was found to be $83.4 \pm 23.5$ and showed no impact or correlation with gender, nationality, weight, height and BMI ,but the age has significant relation with the angle at $p$ value 0.05 table[3,5].A new equation was created to predict the confluence angle when the patients were Asians and their ages are known as presented in the caption below table [6].Figure [1] reflects that as the age increased the confluence angle will be decreased, this presented results are reverse to what was mentioned by Nuray et al, that the age has no impact on the confluence angle [2].

Table [7] presented that the confluence angle did not affect by the biliary tree situation;if it is dilated or if it is in normal size because the $p$ value is 0.376 .

In order to study if there are changes in the angle when the right and left hepatic ducts are dilated, another group of 28 patients were enrolled and were assessed for their demographic data as well as nationality and MRCP findings as seen in table [8].The mean angle was found to be $79.1 \pm$ 20.2 with no significant relations between the variables as presented in tables [9-12]

When examining the confluence angle with dilated and normal ducts within two groups with similar MR associations as gall stone, distended gall bladder, cholecystectomy, normal biliary tree findings; the study showed no significant difference between the confluence angle in all these cases. The study reflects the maximum and minimum values of the confluence angle; it was found to be maximum (105.7, $134.8)$ and minimum $(63.8,38.8)$ when the bile ducts were dilated and maximum (142.2, 155.6) and minimum (36.4, 38.1) when the bile ducts were normal for males and females respectively. Previous studies mentioned that the width of confluence angle may provide an information about the presence of hilar masses, parenchymal atrophy of the liver and periportal space lesions if there is an alteration in the angle on MRCP studies [2].In our study; It had been noticed that the angle is greater when the biliary tree is not dilated but the wide range between the maximum and minimum angles values in the two groups may not allow the dependency upon the angle in diagnosing or prediction of diseases, this was similar to what was mentioned by Nuray et al[2].

The predominant ethnic race in our study population was the Saudi (65\% and 71.4\%) for normal and dilated ducts as presented in table $[4,11]$.Studies postulated that biliary tree might be related to the patient's race and height [12].The biliary confluence angle has no significant relation with the situation of the biliary tree or the association with different MRCP findings as seen in tables [13, 14]

The study concluded that MRCP can evaluate and assess the biliray confluence angle in dilated and non dilated ducts. MR images can diagnose the underlying causes. Dependency upon this angle is not benifectual for diagnosis of prediction of diseases. No significant relation was found between the genders, weight, height, BMI, race, biliary system diseases and the biliary confluence angle but a significant relation was detected with the age. A new equation for angle prediction was established.

\section{Acknowledgements}

We sincerely thank the participants without whom the study would not have been feasible. The Sudan University of Science and Technology- College of Medical Radiological Science Khartoum-Sudan and the Radiology Department-Dr. Soliman Fakeeh Hospital-Al-Medina,Qurban, Saudi Arabia.

\section{References}

[1] LauaM.Fayad,ThomasKowalski,DonaldG.Mitchell,2004.M $\mathrm{R}$ cholangiopancreatography: evaluation of common pancreatic diseases Magn Reson Imaging Clin N Am 12 651-668.

[2] Nuray Haliloğlu, Ayşe Erden, İlhan Erden 2009. Normal Biliary Confluence Angle In Classical Junction Type: Assessment With MR CholangiopancreatographyAnkara Üniversitesi Tıp Fakültesi Mecmuası, 62(4)163-165.

[3] Couinaud C. 1999. Liver anatomy: portal (and supra hepatic) or biliary segmentation. Dig Surg 16:459-467.

[4] Kitami M, Takase K, Murakami G, Ko S,Tsuboi M, Saito H, Higano S, Nakajima Y,Takahashi S. 2006. Types and frequencies of biliary tract variations associated with a major portal venous anomaly: analysis with multi detector row CT cholangiography. Radiology 238:156-166.

[5] Brant PE, Kopke-Aguiar L, Shigueoka DC, et al. 2008.Anicteric cholangiopathy in schistosomiasis patients.Acta Trop.;108:218-21.

[6] Cheng YF, Huang TL, Chen CL, Chen YS,Lee TY. 1997. Variations of the intrahepatic bile ducts: application in living related liver transplantation and splitting liver transplantation. Clin Transplant 11:337-340.

[7] Ohkubo M, Nagino M, Kamiya J, Yuasa N,Oda K, Arai T, Nishio H, Nimura Y. 2004. Surgical anatomy of the bile ducts at the hepatic hilum as applied to living donor liver transplantation. Ann Surg 239: 82-86.

[8] Choi JW, Kim TK, Kim KW, Kim AY, Kim PN,Ha HK, Lee MG. 2003. Anatomic variation in intrahepatic bile ducts: an analysis of intraoperative cholangiograms in 300 consecutive donors for living donor liver transplantation. Korean J Radiol $4: 85-90$ 
[9] Lee VS, Krinsky GA, Nazzaro CA, Chang JS,Babb JS, Lin JC, Morgan GR, Teperman LW. 2004. Defining intrahepatic biliary anatomy in living liver transplant donor candidates at Mangafodipir Trisodium- enhanced MR cholangiography versus conventional T2- weighted MR cholangiography. Radiology233:659-666.

[10] Baillie J, Paulson EK, Vitellas KM. 2003, Biliary imaging: a review. Gastroenterol Clin N Am; 124:1686-99
[11] Wallner B, Schumacher K, Weidenmaier W, et al. 1991.Dilated biliary tract: evaluation with MR cholangiography with a $\mathrm{T} 2$-weighted contrast-enhanced fast sequence. Radiology; 181:805-8

[12] Chuah K B, Yap C K, Ng H S, 2001 .Extra hepatic Bile Duct Length in the Singapore Population, Singapore Med J Vol 42(4) : 165-169. 